ARTÍCULO ORIGINAL

\title{
Indicadores de eficiencia productiva de un módulo experimental de chinchillas y su comparación con los de sistemas de producción comercial
}

\author{
Nistal $\mathrm{AJ}^{1,3,{ }^{*}}$, Zapata $\mathrm{M}^{1,3}$, Mazufero $\mathrm{K}^{3}$, Irazuzta $\mathrm{R}$, Di Masso $\mathrm{RJ}^{2,4}$ \\ ${ }^{1}$ Cátedra de Fisiología - Facultad de Ciencias Veterinarias - Universidad Nacional de Rosario (UNR) \\ ${ }^{2}$ Cátedra de Genética - Facultad de Ciencias Veterinarias - UNR \\ ${ }^{3}$ Módulo Productivo de Chinchillas, Escuela Agrotécnica Libertador General José de San Martín, UNR \\ ${ }^{4}$ Carrera del Investigador Científico - UNR
}

* Correspondencia: Alejandro Nistal, Facultad de Ciencias Veterinarias, Universidad Nacional de Rosario. Ov. Lagos y Ruta 33. 2170 Casilda, Santa Fe, Argentina.

E-mail: anistal@unr.edu.ar

Recibido: 10 Abril 2017. Aceptado: 21 Junio 2017. Disponible en línea: 28 Junio 2017

Editor: C. Baravalle

\begin{abstract}
RESUMEN. En un sistema de producción intensivo de chinchillas, de pequeña escala, manejado en base a un protocolo experimental con control artificial del fotoperíodo se relevaron durante tres años consecutivos 24 indicadores productivo-reproductivos. Los valores de todos los indicadores fueron similares o superiores a los informados en la bibliografía en diferentes sistemas productivos. Ello puede explicarse por: la fertilidad promedio mantenida durante el período estudiado, generada en parte por el refugo sistemático anual de toda hembra sin parto registrado, independientemente de su fenotipo peletero; el tamaño de la camada al nacimiento y al destete, que si bien mostraron valores dentro del rango de referencia bibliográfica, estuvieron acompañados por un porcentaje de mortalidad total satisfactorio y partos por reproductora por año y por madre por año por encima de la mayor parte de los datos referenciados en la bibliografía atribuibles a la estimulación determinada por el manejo fotoperiódico. La conjunción de estos indicadores permitió alcanzar valores de 2,31 y 2,85 gazapos destetados por reproductora y por madre por año. Los valores informados en el Módulo experimental superan en un gazapo destetado por madre y por año a los emprendimientos dedicados a la producción de chinchillas del área de influencia de la FCV-UNR.
\end{abstract}

SUMMARY. Indicators of productive efficiency of an experimental module of chinchillas and comparisons with commercial systems. In a small-scale chinchilla intensive production system, managed on the basis of an experimental protocol with artificial control of the photoperiod, 24 productive-reproductive indicators were surveyed for three consecutive years. The values of all indicators were similar or higher than those reported in the literature in different production systems. This can be explained by: (1) the average fertility maintained during the period studied, generated in part by the annual systematic scrap of all females without registered birth, regardless of their skin phenotype; (2) the size of litter at birth and weaning, which although showed values within the range of bibliographic reference, were accompanied by an acceptable percentage of total mortality, and (3) births per reproductive female per year and per mother per year above most of the data referenced in the literature, attributable to the stimulation determined by photoperiodic management. The values reported in the Experimental Module surpassed in one kitten weaned per mother and per year, the enterprises dedicated to the production of chinchillas of the area of influence of the FCV-UNR.

Palabras clave: Reproducción, fertilidad, prolificidad, mortalidad, Chinchilla lanigera.

Key words: Reproduction, fertility, prolificacy, mortality, Chinchilla lanigera

\section{Introducción}

La chinchilla (Chinchilla lanigera) es un pequeño mamífero, herbívoro y de hábitos nocturnos. Pertenece a uno de los géneros de roedores autóctonos de la Cordillera de los Andes y, dada su condición de recurso peletero, la caza indiscriminada la ha llevado prácticamente a su extinción en vida libre (Spotorno, 2004; Valladares Faúndez et al., 2014).
Es una especie poliéstrica estacional (Burgess y Bishop, 2012), cuya temporada de servicios en vida libre se presenta de mayo a noviembre (Neira et al., 1989). Posee un ciclo estral prolongado en comparación con la mayoría de los animales domésticos. Algunos autores informan una duración de 41 días, con el $75 \%$ de los ciclos de 30 a 60 días (Weir, 1970). Otros (Spotorno et al., 2004) reportan valores de 38,1 $\pm 0,7$ días con un rango entre 16 y 69 días. El estro tiene una duración promedio de 48 horas con la capacidad de presentar un celo fértil posparto, ocurriendo la ovulación entre 30 y 36 horas luego del parto (Weir, 1970; Spotorno et 
al., 2004). De no presentarse este celo, o de no producirse la fecundación y consecuente gestación, el próximo celo ocurre luego del anestro lactacional, que dura $56 \pm 12$ días para lactancias clásicas de dos meses aproximadamente (Weir, 1970). Presenta un período de gestación también largo, con una media de 111 días y un rango entre 105 y 118 días (Weir, 1970; Grau, 1993; Bronson, 1999; Spotorno et al., 2004). Si bien las hembras pueden producir hasta tres camadas en el año, se informan valores que no superan los 2 partos por hembra total del plantel, con un periodo interparto de 165-231 días (Der Deutsche Pelztierzuchter, 1987; Neira et al., 1989; García Márquez y García Márquez, 2004; Brzozowski y Nyrek-Koczkodaj, 2007).

Argentina ha sido, tradicionalmente, un importante exportador de "pieles" producidas a partir de animales criados en cautiverio. Los emprendimientos productivos de este tipo, localizados en el sur de la provincia de Santa Fe y oeste de Córdoba, presentan una matriz micronizada, con establecimientos de escala reducida instalados como alternativa laboral secundaria a la principal actividad económica familiar, sin relación con otras producciones agropecuarias, atendidos por sus propios dueños, emplazados en el ejido urbano y con elevado nivel de rotación de productores. En general, los mismos presentan bajo desempeño productivo con un valor promedio de 1,7 gazapos destetados por madre al año (Nistal et al., 2005), hecho asociado a las inapropiadas condiciones generales de control ambiental (manejo con fotoperíodo natural, escaso control de temperatura y humedad), alimentación poco adecuada (variación del aporte de nutrientes por alternar raciones de alimentos voluminosos y concentrados) y a cierta resistencia a eliminar del plantel de reproductores a los animales infértiles si los mismos presentan un buen fenotipo peletero. Esta conducta está determinada, al menos en parte, por la carencia de información de base científica por parte de los productores, la falta de intervención técnica profesional especializada, el predominio de canales no formales de información y prácticas de manejo general con fundamentación empírica (Nistal y Di Masso, 2009b).

En octubre de 2011 se crea el Módulo Productivo de Chinchillas de la Escuela Agrotécnica Libertador General José de San Martín, dependiente de la UNR, con el objetivo de desarrollar actividades de docencia e investigación sobre aspectos relacionados con el manejo general y productivo de esta especie en cautiverio. El objetivo de este trabajo fue describir los valores de algunos indicadores productivo-reproductivos registrados en dicho Módulo durante tres años consecutivos y compararlos con los valores de referencia informados en la literatura y con algunos registros provenientes de explotaciones comerciales ubicadas en el área de influencia de la Facultad de Ciencias Veterinarias de la UNR.

\section{Materiales y Métodos}

La información evaluada en este trabajo se generó en el Módulo Productivo de Chinchillas de la Escuela Agrotécnica Libertador General José de San Martín de la UNR. El mismo se fundó con 36 reproductores vírgenes. Inicialmente el plantel se conformó con seis familias poligámicas, cuatro de ellas constituidas por seis hembras y un macho de pelaje estándar y dos familias "mutación", una negra y otra beige, de tres hembras cada una con un macho. Los reproductores del plantel fundador ingresaron con una edad promedio de $12,3 \pm 2,32$ meses y un peso corporal promedio de $704 \pm 71,2 \mathrm{~g}$ en el caso de las hembras y de 13,6 \pm 4,15 meses y $641 \pm 81,4 \mathrm{~g}$ en el caso de los machos.

Se trata de un sistema de producción animal intensivo de pequeña escala manejado en base a un protocolo experimental (Nistal y Di Masso, 2009a). La nave de cría presenta paredes y techo de mampostería, piso recubierto de baldosas, cobertura sobre todas las paredes y techo que la protege del impacto del sol, control de temperatura $\left(16^{\circ}-24^{\circ}\right)$ y humedad (inferior a 70\%) ambiente y circulación de aire (equipo acondicionador y extractor de aire). Los animales se alojan en jaulas individuales de $30 \mathrm{~cm}$ de ancho $x 30 \mathrm{~cm}$ de alto $x$ $45 \mathrm{~cm}$ de profundidad provistas con un piso constituido por una bandeja metálica de acero inoxidable cubierta con una cama mineral (piedras sanitarias) de recambio quincenal. Los animales se mantienen bajo un manejo fotoperiódico artificial, con anulación de la luz natural mediante el oscurecimiento de puerta y ventanas y su reemplazo por luz artificial. Esta última es suministrada por seis tubos fluorescentes de $36 \mathrm{~W}$ cada uno, que generan una densidad luminosa incidente de 25 a 250 lux con una temperatura de color de más de $5000 \mathrm{~K}$. El manejo lumínico consta de dos fases de ascenso fotoperiódico, de 10:00 a 13.30 horas de luz/día, con un incremento diario de 1:24 minutos, de cinco meses cada una (del 1 de diciembre al 30 de abril la primera y del 1 de junio al 31 de octubre la segunda) con un corto período de descenso fotoperiódico brusco a 10 horas de luz/día, de un mes de duración, intercalado en los meses de mayo y noviembre. La alimentación de los animales consiste en alimento balanceado, en pellets, con $17 \%$ de proteína bruta, $3 \%$ de grasa, $18 \%$ de fibra, $14 \%$ de humedad, $1 \%$ de calcio y $0,5 \%$ de fósforo, complementada con fardo o cubos de alfalfa (Medicago sativa) de muy buena calidad. Ambos tipos de alimentos (concentrados y voluminosos) se administran diariamente ad libitum.

Durante los primeros tres años se estimaron los valores de los siguientes 24 indicadores productivoreproductivos:

1. Reproductoras (R) - Número de hembras que conforman el plantel de reproducción.

2. Madres (M) - Número de reproductoras que parieron en el año de estudio. 
3. Fertilidad (F) - Proporción de madres en relación a las reproductoras $[\mathrm{F}=\mathrm{M} / \mathrm{R} \times 100]$.

4. Partos $(P)$ - Número total de partos que tuvieron las madres en el año de estudio.

5. Partos de camada de un gazapo (P1) - Proporción de los partos totales correspondientes a camadas de un único gazapo.

6. Partos de camada de dos gazapos (P2) - Proporción de los partos totales correspondientes a camadas de dos gazapos.

7. Partos de camada de tres gazapos (P3) - Proporción de los partos totales correspondientes a camadas de tres gazapos.

8. Partos de camada de cuatro o más gazapos $(P 4 \circ+)$ Proporción de los partos totales correspondientes a camadas de cuatro o más gazapos.

9. Gazapos nacidos (N) - Número total de gazapos nacidos en el año.

10. Tamaño de camada al nacimiento (TCN) - Índice que relaciona el número de gazapos nacidos y el número total de partos $[\mathrm{TCN}=\mathrm{N} / \mathrm{P}]$

11. Gazapos nacidos vivos (NV) - Número total de gazapos nacidos que al momento de su primera inspección (dentro de las 24 horas de su nacimiento) se hallaron con vida.

12. Gazapos nacidos vivos por parto (NVP) - Índice que relaciona el número de gazapos nacidos vivos y la cantidad total de partos [NVP $=\mathrm{NV} / \mathrm{P}]$.

13. Gazapos nacidos muertos (NM) - Número total de gazapos nacidos que al momento de su primera inspección (dentro de las 24 horas de nacimiento) se hallaron sin vida (crías nacidas muertas a término, fetos frescos, momificados, abortados y malformados).

14. Porcentaje de mortalidad al nacimiento (PMN) Proporción de gazapos nacidos muertos en relación al total de gazapos nacidos $[\mathrm{PMN}=\mathrm{NM} / \mathrm{N}]$.

15. Gazapos muertos en lactancia (ML) - Número de gazapos nacidos vivos y muertos durante su lactancia.

16. Porcentaje de mortalidad en lactancia (PML) Proporción de gazapos muertos en lactancia en relación al total de nacidos vivos $[\mathrm{PML}=\mathrm{ML} / \mathrm{NV} \times 100]$.

17. Gazapos destetados (D) - Número de gazapos destetados con vida en el año de estudio.

18. Tamaño de camada al destete (TCD) - Índice que relaciona el número total de gazapos destetados y el número total de partos $[T C D=D / P]$.
19. Partos por reproductora por año (PRA) - Índice que relaciona la cantidad de total de partos y la cantidad de hembras reproductoras $[P A R=N P / R]$.

20. Partos por madre por año (PMA) - Índice que relaciona la cantidad total de partos y la cantidad de madres $[\mathrm{PMA}=\mathrm{NP} / \mathrm{M}]$.

21. Gazapos nacidos por reproductora por año (GNRA) - Índice que relaciona la cantidad de gazapos nacidos y la cantidad de hembras reproductoras [GNRA = N/R] .

22. Gazapos nacidos por madre por año (GNMA) Índice que relaciona la cantidad de gazapos nacidos y la cantidad de madres [GNMA $=\mathrm{N} / \mathrm{M}$ ].

23. Gazapos destetados por reproductora por año (GDRA) - Índice que relaciona la cantidad de gazapos destetados y la cantidad de hembras reproductoras [GDRA = D/R].

24. Gazapos destetados por madre por año (GDMA) Índice que relaciona la cantidad de gazapos destetados y la cantidad de madres [GDMA = D/M]

Los valores de estos indicadores se compararon, a nivel descriptivo, con valores publicados provenientes de establecimientos localizados en diversos países con mayor o menor tradición en este tipo de producción, como así también con valores relevados en criaderos de la zona de influencia de la FCV-UNR. Cabe mencionar que las características de la nave y el control de las variables ambientales, como así también el tamaño, material, forma y agrupamiento de las jaulas poseen similares patrones generales que los informadas por diferentes autores para criaderos de diversas procedencias (Aleandri, 2000; Grau, 1993; Neira y col., 1989; García Ferrada y col., 1991; Seremak y Sulik, 2003; Socha y Olechno, 2000; Felska-Błaszczyk y Brzozowski, 2005; Poyraz y col., 2005; Kołodziejczyk y col, 2014; Wojtacka y col., 2014) lo que pone de manifiesto que, al igual que en otras explotaciones intensivas, en el caso de la cría de chinchillas también se replican de país en país la cuestiones básicas asociadas a la estructura de producción

\section{Resultados}

La Tabla 1 resume los valores de los indicadores de eficiencia productivo-reproductiva relevados en el Módulo durante tres años consecutivos conjuntamente con los valores totales y promedio del trienio. Tanto el número de hembras reproductoras como el número de madres se mantuvieron constantes en los tres años considerados. Los indicadores relevados presentaron oscilaciones entre años. El segundo año se caracterizó por una disminución del número de partos, con un aumento en la proporción de partos con un único gazapo en detrimento de las categorías restantes lo que 
se tradujo en un menor número de gazapos nacidos, en una reducción del tamaño promedio de la camada al nacimiento, en el porcentaje de mortalidad al nacimiento y en aquellos indicadores relativizados por el número de madres o de reproductoras por madre y por año. En el tercer año se registró una mayor mortalidad porcentual durante la lactancia con repercusión sobre los gazapos destetados y el tamaño de la camada al destete.

Tabla 1: Indicadores de eficiencia productivo-reproductiva correspondientes al Módulo Productivo de Chinchillas de la Escuela Agrotécnica Libertador General José de San Martín, Universidad Nacional de Rosario

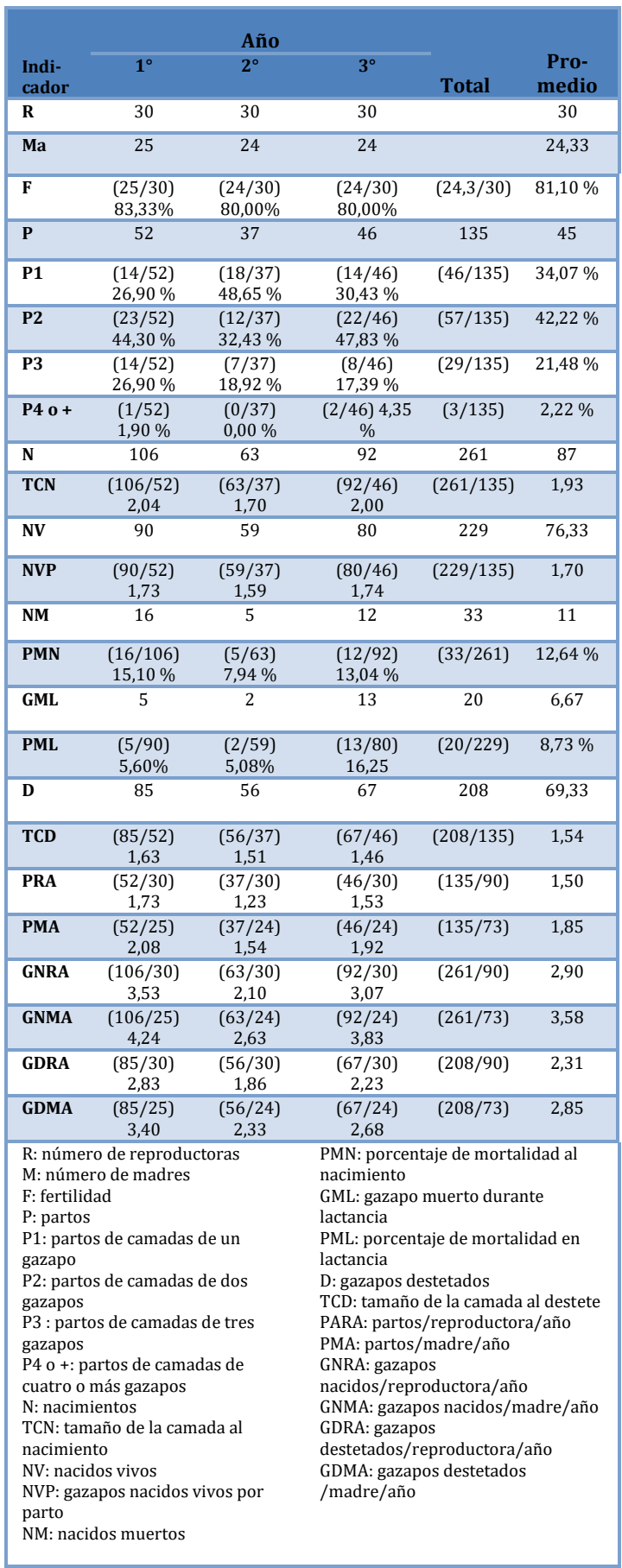

\section{Discusión}

El valor de fertilidad promedio del $81 \%$ registrado en nuestro Módulo coincidió con el límite inferior de los rangos del $80-90 \%$ y del $80-85 \%$ mencionados por Cornett y Cornett (1984) y en Der Deutsche Pelztierzuchter (1987), respectivamente, y con el límite superior del rango del $75-82 \%$ publicado por Seremak y Sulik (2003), fue igual al $80,9 \%$ mencionado por Neira et al. (1989), se ubicó en el punto medio del intervalo del 75 al $87 \%$ observado por Busso et al. (2012) y superó los registrados por Álvarez Aro (2010) que oscilaron entre $63-75 \%$.

El tamaño de la camada al nacimiento de 1,93 gazapos, promedio para los tres años de estudio, superó los valores entre 1,70-1,75 citados por Morales et al. (1997) y por Neira et al. (1989) en explotaciones comerciales de Chile y se ubicó dentro de los valores de referencia de 1,75 a 2,19 mencionados en EE.UU. (Cornett y Cornett, 1984), Europa (Der Deutsche Pelztierzuchter, 1987; Felska-Błaszczyk y Brzozowski, 2005; Socha et al., 2009; Dzierżanowska-Góryń et al., 2011; 2014) y Asia (Kuroiwa y Imamichi, 1977). El valor de ese indicador está determinado por la proporción de partos simples (34\%), dobles (42\%), triples (21\%) y de aquellos que involucraron a cuatro o más crías (3\%). En el año con menor tamaño de la camada al nacimiento se observó una mayor proporción de partos simples seguida por partos dobles y triples, comportamiento similar al informado por Neira et al. (1989), lo que determinaría el menor valor de TCN en estos establecimientos.

En los dos años restantes predominaron los partos dobles, presentándose incluso partos de cuatro y seis gazapos situación coincidente con la informada por autores europeos (Dzierżanowska-Góryń et al., 2014) y asiáticos (Kuroiwa y Imamichi, 1977) con valores generales más altos de TCN.

El tamaño promedio de la camada al destete, resultante de los efectos conjuntos del tamaño de la camada al nacimiento y la mortalidad durante la lactancia, fue de 1,54 gazapos valor que se encuentra dentro del rango de 1,31-1,93 gazapos citado en la bibliografía (Kuroiwa y Imamichi, 1977; Neira et al., 1989; Morales et al. 1997; Felska-Błaszczyk y Brzozowski, 2005; Socha et al., 2009; DzierżanowskaGóryń et al., 2011; 2014).

La mortalidad porcentual al nacimiento del $12,6 \%$ es consistente con los valores de 7 a $16 \%$ mencionados por Neira et al. (1989) y por García Ferrada et al. (1989). Por su parte, el $9 \%$ de mortalidad durante la lactancia se encuentra muy por debajo del $21-28 \%$ mencionado en establecimientos chilenos (Neira et al., 1989; García Ferrada et al., 1989; Álvarez Aro, 2010) y del $15 \%$ informado por diversos autores para criaderos de EE.UU. y Europa (Cornett y Cornett, 1984; Der Deutsche Pelztierzuchter, 1987). 
Cuando se considera el $21 \%$ de pérdidas contabilizadas en el Módulo por mortalidad acumulada en el período nacimiento-destete, las mismas fueron inferiores al rango del $25-38 \%$ informado por algunos autores (Álvarez Aro, 2010; Cornett y Cornett, 1984; Der Deutsche Pelztierzuchter, 1987; Neira et al., 1989; García Ferrada et al., 1989), coincidentes con valores cercanos al $20 \%$ reportados por Seremak y Sulik (2003), Seremak (2007), Felska-Błaszczyk et al. (2008) y Dzierżanowska-Góryń et al. (2014) y superiores al rango $13,5 \%-16,5 \%$ consignados por Felska-Błaszczyk y Brzozowski (2004; 2005) lo que implica, que si bien es un valor aceptable, también puede ser optimizado.

Un carácter de trascendencia relacionado con el tamaño de la camada es el peso del gazapo al nacimiento. El valor promedio de esta variable en el Módulo fue de $51 \mathrm{~g}$, superó los valores de 43 y $44 \mathrm{~g}$ informados por Weir (1970) y Bickel (1983), respectivamente y coincidió, aproximadamente, con el límite superior del intervalo entre 40 y $50 \mathrm{~g}$ publicado por Grau (1993) y con el valor promedio de $49,5 \mathrm{~g}$ de Dzierżanowska-Góryń et al. (2014) y de 52 g de Neira (1893).

Las variables tamaño de camada y mortalidad al nacimiento estuvieron estrechamente relacionadas. $\mathrm{EI}$ aumento del tamaño de la camada afecta negativamente el peso corporal individual de los gazapos y la disminución del peso corporal aumenta la mortalidad (Neira et al., 1989; Spotorno et al., 2004; Barabasz y Łapiński, 2008; Dzierżanowska-Góryń et al., 2014). A menor tamaño de la camada gestada el mayor espacio disponible por gazapo y la mejor nutrición uterina relativa derivada de una menor competencia permite a los fetos expresar más plenamente su capacidad de crecimiento en un ambiente con menores restricciones, alcanzar un mayor peso al nacimiento $y$ mostrar mayor vigor durante la lactancia. A este respecto cabe consignar que no nacieron gazapos vivos con un peso inferior a $29 \mathrm{~g}$, como tampoco se destetaron gazapos con un peso al nacimiento inferior a $30 \mathrm{~g}$. El peso promedio de $41 \mathrm{~g}$ registrado en los natimortos fue inferior en $11 \mathrm{~g}$ al de los gazapos nacidos vivos que promediaron los $53 \mathrm{~g}$. De la misma manera el peso promedio al nacimiento de $54 \mathrm{~g}$ de los gazapos finalmente destetados fue superior al de los gazapos muertos durante la lactancia (50 g).

Las muertes de gazapos durante la lactancia ocurrieron, en la mayoría de los casos, a los pocos días de nacidos, como consecuencia de su bajo peso al nacimiento y al bajo aumento de peso diario puesto de manifiesto. Este comportamiento coincide con lo informado por diferentes autores (Lanszki, 1996; Seremak, 2007; Barabasz y Łapiński, 2008; DzierżanowskaGóryń et al., 2014) que consignan que más del $90 \%$ de las muertes que tienen lugar durante la lactancia ocurren en las dos primeras semanas de vida, considerando a estos quince días iniciales como un período crítico para la supervivencia de las crías, caracterizado por una alta tasa de mortalidad asociada a la debilidad de las mismas atribuible a su bajo peso al nacimiento, a una escasa producción láctea de la madre o a la competencia entre hermanos en el caso de camadas numerosas como ocurrió ante pariciones de camadas con seis integrantes observadas en el tercer año. Este año se caracterizó por un aumento de la mortalidad asociada a una disminución del peso corporal como consecuencia del aumento del tamaño de camada al nacimiento, en tanto se presentaron partos cuádruples y séxtuples con un peso promedio de $29 \mathrm{~g}$ en el caso de los gazapos provenientes de camada de seis integrantes y una mortalidad al destete del $60 \%$ para ese tamaño de camada.

En pocos casos la pérdida de gazapos tuvo lugar más allá de los 15 días de vida y, en todos los casos observados, la causa fue la agresión de otra hembra reproductora de la familia cuando el gazapo se escapó de su jaula e ingresó a otra por pérdida de la tapa que obtura la tronera.

Tanto los valores promedio del número de partos por reproductora por año como del número de partos por madre por año - 1,50 y 1,85 respectivamente - superan el límite superior de los rangos de 1,18-1,34 para el primero y de 1,48-1,62 para el segundo, correspondientes a explotaciones de América y Europa (Cornett y Cornett, 1984; Der Deutsche Pelztierzuchter, 1987; Neira et al., 1989; Felska-Błaszczyk y Brzozowski, 2005; Dzierżanowska-Góryń et al., 2014), si bien Dzierżanowska-Góryń et al. (2011) informaron valores de 2,03 partos por madre por año en un establecimiento de producción y selección comercial polaco.

Los resultados productivo-reproductivos obtenidos bajo este protocolo de producción se pueden considerar como muy buenos dado que los valores de todos los indicadores fueron similares o superiores a los informados en los diferentes sistemas productivos citados. El buen desempeño observado en este aspecto en el Módulo se puede explicar principalmente por: (a) la fertilidad promedio mantenida durante el período estudiado, generada en parte por el refugo sistemático al final de cada ciclo anual de todas las hembras sin parto registrado, independientemente de su fenotipo peletero, (b) el tamaño de la camada al nacimiento $\mathrm{y}$ al destete, indicadores que si bien mostraron valores dentro del rango de referencia bibliográfica, estuvieron acompañados por un porcentaje de mortalidad total en el período nacimientodestete satisfactorio, posiblemente debido al mantenimiento de un ambiente homogéneo en términos de manejo de la alimentación y control de la temperatura, humedad y fotoperíodo) y (c) partos por reproductora por año y partos por madre por año con valores por encima de la mayor parte de los datos referenciados en la bibliografía consultada, atribuibles al menos parcialmente, a la estimulación determinada por el manejo fotoperiódico ensayado (Nistal y Di 
Masso, 2008, 2011). La conjunción de estos indicadores permitió alcanzar valores de 2,31 y 2,85 gazapos destetados por reproductora y por madre por año, respectivamente, posicionándose por encima de los valores de 1,$85 ; 2,16$ y 2,26 informados por Seremal y Sulik (2003), Felska-Błaszczyk y Brzozowski (2005) y Dzierżanowska-Góryń et al. (2014); en coincidencia con el rango entre 2,22 - 2,79 publicado por Kołodziejczyk et al. (2014) y por debajo de los valores informados por Dzierżanowska-Góryń et al. (2011) en un establecimiento modelo en Polonia con un rango de 1,23-3,56 crías destetadas por hembra y año que, si bien extraordinarios en el caso del límite superior del intervalo, ponen en evidencia la potencialidad reproductiva de la especie. En relación a la producción de los establecimientos de la zona, los valores informados en el Módulo experimental superan en un gazapo destetado por madre y por año (80\% superior) a los emprendimientos dedicados a la producción de chinchillas del área de influencia de la Facultad de Ciencias Veterinarias de la Universidad Nacional de Rosario (Nistal y Di Masso, 2009), por lo que el protocolo de trabajo propuesto permite sugerir su implementación con el fin mejorar el desempeño de estos sistemas comerciales poniendo énfasis en la utilización de un manejo fotoperiódico caracterizado por dos fases ascendentes en el año (estimulación reproductiva de una especie estacional con mayor actividad en días crecientes), homogeneización del ambiente caracterizado por el aporte ad libitum de alimento balanceado y heno, estabilización de la temperatura, disminución de la humedad y aumento de la ventilación dentro de la habitación de cría, sumado al refugo estricto de animales sub e infértiles.

\section{Bibliografía}

Aleandri F. 2000. Cría y comercialización de la Chinchilla. 2da ed. Buenos Aires: ABRN- Producciones Gráficas SRL. pp. 4175.

Álvarez Aro ML. 2010. Manejo y crianza de la Chinchilla lanigera para la obtención de pieles en la Región de Magallanes. Tesina de titulación de Ingeniero de Ejecución Agropecuario. Universidad de Magallanes. Disponible en: http://www.umag.cl/biblioteca/tesis/alvarez_aro_2010.pdf

Barabasz B, Lapiński S. 2008. Growth rate of sucking chinchilla pups and lactating performance of their dams. Anim. Sci. Pap. Rep. 26: 227-234.

Bronson FH. 1999. Rodentia. In Knobil E, Neill JD (eds): Encyclopedia of reproduction. Academic Press, 282-9, New York, EE.UU. pp. 282-289.

Brzozowski M, Nyrek-Koczkodaj A. 2007. Chinchillas reproduction results in relation to the age of the first mating time. Ann. Warsaw Univ. of Life Sci.-SGGW, Anim. Sci. 44: 3-7.

Burgess \& Bishop. 2012. Reproductive Physiology, Normal Neonatology, and Neonatal Disorders of Chinchillas, in Management of Pregnant and Neonatal Dogs, Cats, and Exotic Pets. Ed. C. Lopate, J Wiley \& Sons, Ltd, Chichester, UK. pp. 295-308.
Busso JM, Ponzio MF, Fiol de Cuneo M, Ruiz RD. 2012. Reproduction in chinchilla (Chinchilla lanigera): Current status of environmental control of gonadal activity and advances in reproductive techniques. Theriogenology 78: 1-11.

Cornett B, Cornett J. 1984. Statistics in the search for a realistic idea of chinchilla production potential. En: Ranchers Handbook, previously known as Chinchilla fur Farming Series, Empress Chinchilla Breeders Cooperative. Inc., Sixes, Oregon. pp. 284-837.

Der Deutsche Pelztierzuchter. 1987. Zuchterfolge bei Cinichillas. Der Deutsche Pelztierzuchter 61: 72.

Dzierżanowska-Góryń D, Brzozowski M, Góral-Radziszewska K. 2014. Young chinchillas weight gain, depending on their body mass at birth. Ann. Warsaw Univ. of Life Sci.-SGGW, Anim. Sci. 53: 95-101.

Dzierżanowska-Góryń D, Góral K, Głogowski R. 2011. The analysis of chinchilla females reproduction (Chinchilla laniger M.), on the example of Polish breeding farm. Ann. Warsaw Univ. of Life Sci.-SGGW, Anim. Sci. 49: 21-26.

Dzierzanowska-Goryn D, Kaleta T, Kowalczyk M. The behaviour and an activity of chinchilla (Chinchilla lanigera) kept under laboratory conditions. Proceeding of the XIIth International Congress on Animal Hygiene ISAH; 2005 Sept 48; Warszaw, Poland; 2: 462-465.

Felska-Błaszczyk L, Brzozowski M. 2004. Litter size, weaning success, and nursing mortality in chinchillas (Chinchilla lanigera) in relation to cage illumination. Scientifur 28: 234237.

Felska-Błaszczyk L, Brzozowski M. 2005. Effect of light intensity on reproduction of Polish, Swedish and Danish chinchillas. Arch. Tierz., Dummerstorf. 48: 494-504.

Felska-Błaszczyk L, Sulik M, Semik A, Seremak B. Polish vs. Danish chinchillas (Chinchilla laniger M.) 2008. An analysis of body conformation and reproduction performance in two populations. Scientifur 32: 42-46.

García Ferrada MX, Neira R, Scheu Hitschfeld RA. 1989. Variación ambiental en características reproductivas en chinchillas (Chinchilla lanigera Gray) en confinamiento. Avances en producción animal. 14: 121-127.

Grau J. 1993. La Chinchilla: su crianza en todos los climas. El Ateneo, Buenos Aires, Argentina. pp. 62-82.

Kołodziejczyk D, Osiński M, Socha S. 2014. Analysis of profitability of chinchilla farming based on the production results of a selected farm. Acta Sci. Pol. Zootech. 13: 55-66.

Kuroiwa J, Imamichi T. 1977. Growth and reproduction of the chinchilla-age at vaginal opening, oestrous cycle, gestation period, litter size, sex ratio, and diseases frequently encountered. Jikken Dobutsu 26: 213-222.

Lanszki J. 1996. The effect of litter size and individual weight at birth on the growth and mortality of chinchillas. Scientifur 20: 42-47.

Morales M, Ibarra M, Viñas L, Briones A. 1997. Indicadores demográficos de fecundidad en chinchilla hembra de criadero. Avances en Ciencias Veterinarias 12: 71-74.

Neira R, García X, Scheu R. 1989. Análisis descriptivo del comportamiento reproductivo y de crecimiento de chinchillas en confinamiento. Avances en Producción Animal 14: 109119. 
Nistal, A.J.; Di Masso, R, J, Catalani, G.; Marconi, A. 2005. Farm size and breeding indixes in chinchilla micro furindustry. Biocell 29: 136.

Nistal AJ, Di Masso RJ. 2008. Indicadores reproductivos y fotoperíodo en Chinchilla lanigera. En: Actas de las IX Jornadas de Divulgación Técnico-Científicas de la Facultad de Ciencias Veterinarias de la Universidad Nacional de Rosario. Agosto de 2008, Casilda, pp. 172-173.

Nistal, A.J.; Di Masso, R.J. 2009a. Estacionalidad reproductiva de la chinchilla (Chinchilla lanigera) en condiciones de cautiverio. XIII Jornadas sobre Producción Animal. Asociación Interprofesional para el Desarrollo Agrario (AIDA) - Zaragoza (España). Vol. 1, págs. 669-671.

Nistal AJ, Di Masso RJ. 2009b. Factores de producción en establecimientos dedicados a la cría de chinchillas (Chinchilla lanigera). Rev. Arg. Prod. Anim. 29: 360-361.

Poyraz O, Akinci Z, Onbasllar EE. 2005. Phenotypic correlations among some traits in Chinchilla lanigera produced in Turkey. Turk. J. Vet. Anim. Sci. 29: 381-384.

Seremak B, Sulik M. 2003. An attempt to determine causes of lowered values of breeding indices on a chinchilla farm. Scientifur 26: 75-78.

Seremak B. 2007. [Selected aspects of chinchilla (Chinchilla laniger M.) reproduction under farm management]. In Polish. Rozprawy 240: 1-60.

Socha S, Olechno A. 2000. Analysis of changebility of features in chinchillas. EJPAU 3:4.

http://www.ejpau.media.pl/volume3/issue2/animal/art04.html.

Socha S, Wójcik D, Konopka E. 2009. The analysis of the factors that influence the fertility in three colour types of chinchilla. Zootehnie şi Biotehnologii 42: 375-378.

Spotorno, AE, Zuleta CA, Valladares JP, Deane, AL, Jiménez JE. 2004. Chinchilla laniger. American Society of Mammologists. Mammalian Species 758: 1-9.

Valladares Faúndez P, Spotorno Oyarzún A, Zuleta Ramos C. 2014. Natural history of the Chinchilla genus (Bennett 1829). Considerations of their ecology, taxonomy and conservation status. Gayana 78: 135-143.

Weir BJ. Chinchilla. In: Hafez, E.S.E. (Ed.), Reproduction and Breeding Techniques for Laboratory Animals. Lea and Febiger, Philadelphia, 1970; pp. 209-223. 